\title{
过碱烷基酚钙与二烷基二硫代磷酸锌复配形成摩擦膜的表征
}

\author{
万勇* 晁闻柳 \\ (青岛理工大学机械工程学院, 山东 青岛 266033)
}

\begin{abstract}
摘要: 利用 $X$ 射线吸收精细结构光谱(XANES)研究了过碱硫化烷基酚钻与二烷基二硫代磷酸锌(ZDDP)复配 使用时对 ZDDP 形成摩擦膜的表面化学行为的影响, 并提出了其和 ZDDP 的相互作用机制. 结果表明, 在 ZDDP 中加人过碱硫化烷基酚钙后完全改变了 ZDDP 单独使用时形成摩擦膜的结构, 摩擦膜中含有一定数量的钻离 子, 其结构以磷酸钙为主; 对较高碱值的过碱硫化烷基酚钙, 在高浓度下使用时, 在摩擦膜形成的同时观测到碳 酸钻颗粒的沉积. 此外, ZDDP 和过碱硫化烷基酚钻共同使用时形成的摩擦膜厚度减小. 摩擦膜特性的改变是 ZDDP 和过碱硫化烷基酚钲复配体系抗磨性能变差的主要原因.
\end{abstract}

关键词：ZDDP；金属清净剂；摩擦膜； XANES

中图分类号：0648

\section{Characterization of Tribofilms from Zinc Dialkyldithiophosphate in the Presence of Overbased Calcium Phenate Detergents}

\author{
WAN Yong * CHAO Wen-Liu \\ (School of Mechanical Engineering, Qingdao Technological University, Qingdao 266033, Shandong Province, P. R. China)
}

\begin{abstract}
The effect of overbased calcium phenate detergents on the chemical composition of tribofilms from zinc dialkyldithiophosphate (ZDDP) under boundary lubrication has been studied using P, S, Ca, and C X-ray absorption near edge structure (XANES) spectroscopy. Boundary lubrication was achieved by a pin-on-disk reciprocating wear machine. Calcium phenate detergents, in both high and low overbased forms, were used in combination with ZDDP. C $K$-edge XANES showed that when higher overbased detergents were used the micelles containing calcium carbonate broke down and calcium carbonate was deposited in the tribofilm. Direct evidence for the formation of calcium phosphate from ZDDP in combination with detergents was provided by XANES spectroscopy at the $P_{-}, K^{-}$and $L$ edges. $\mathrm{Zn}^{2+}$ ions were replaced by $\mathrm{Ca}^{2+}$ in the polyphosphate structure of tribofilms forming short chain polyphosphates. The effects of these chemical changes were correlated to the tribological performance of ZDDP/detergents systems.
\end{abstract}

Key Words: ZDDP; Detergent; Tribofilm; XANES

二烷基二硫代磷酸锌(ZDDP)兼有抗氧、抗磨等 功能, 加上其生产成本低廉, 自上世纪中叶问世以来 一直是内燃机油等油品中不可缺少的添加组分 [1]. 而现代内燃机油中, 除 ZDDP 外还含有超过十种的 其他添加剂, 其中用量最大的为金属清净剂, 主要包 括磺酸盐、硫化烷基酚盐、烷基水杨酸盐、环烷酸盐、 硫磷酸盐及其他羧酸盐, 均为典型的表面活性剂, 用 来抑制或减少各种内燃机油沉积物的生成, 延长油
品使用寿命. 目前主要使用高碱值金属清净剂, 即将 碳酸盐和氢氧化物的超细粒子分散在中性的金属清 净剂中制备而得[1.

研究表明, ZDDP 抗磨作用机制在于其在摩擦 过程中与表面反应生成的一层含有硫、磷和锌等元 素的有机/无机化合物摩擦膜, 这层膜具有较低的剪 切强度, 阻止了摩擦副的接触, 从而降低了材料的摩 擦和磨损 ${ }^{[2,3]}$. 然而, 人们也发现金属清净剂严重影响

Received: August 14, 2008; Revised: September 18, 2008; Published on Web: November 7, 2008.

*Corresponding author. Email: wanyong@qtech.edu.cn

(C) Editorial office of Acta Physico-Chimica Sinica 
ZDDP 的抗磨性能. 目前对此存在着两种解释, 一种 理论认为, 金属清净剂和 ZDDP 竞争吸附在表面活 性位, 从而降低了表面 ZDDP 吸附的有效浓度; 另 一种理论则把金属清净剂和 ZDDP 的相互作用归 结为高碱性金属清净剂阻止了 ZDDP 分解产生的 酸性中间产物.上述两种过程均可影响摩擦过程中 ZDDP 在金属表面形成的摩擦膜.

过去, 人们曾利用不同的现代表面分析工具研 究摩擦过程中 ZDDP 形成的摩擦膜的结构 ${ }^{[4-8]}$, 其中 $X$ 射线吸收精细结构光谱 (XANES) 具有明显的优 势, 通过总电子产量(total electron yield, TEY)和苂 光产量(fluorescence yield, FY)模式的测量, 可以分 别得到表面/近表面膜(约 $5 \mathrm{~nm}$ )和本体(约 $50 \mathrm{~nm}$ )元 素的详细化学状态. 此外, 利用高强度的同步加速器 辐射可以在几分钟内收集数据, 而且同步加速器具 有很广的光谱范围, 并能产生能量可变的光子, 因 此 XANES 对小原子的位移结合键和键角相对的 原子位置等非常敏感, 可以获得更为细致的表面信 息 ${ }^{[9]}$. Kasrai 等人 ${ }^{[10-15]}$ 首先利用 XANES 对 ZDDP 以 及 ZDDP 和其他添加剂的复配体系形成的摩擦膜 结构进行了系统的研究. 最近, 任天辉等人 ${ }^{[16-18]}$ 采用 XANES 分析了含硫化合物抗磨膜和热膜的化学特 性. Wan 等 ${ }^{[19]}$ 也曾用 XANES 研究了过碱硫化烷基 酚钲(简称酚钙)单独使用时形成摩擦膜的结构. 本 文利用 XANES 研究了酚钙和 ZDDP 复配使用时形 成摩擦膜的表面化学行为, 并提出了酚钙和 ZDDP 的相互作用机制.

\section{1 实验部分}

\section{1 原材料}

本文所用的添加剂均为 Esso Canada 公司生 产的商业产品, 使用时未经进一步纯化. ZDDP 是 由含 $85 \%$ 的二级醇和 $15 \%$ 的一级醇制备而得, 金 属清净剂为具有不同总碱值 (total base number, TBN)的酚钙, TBN 为 135 和 250 , 分别表示为酚钙1 和酚钙-2. 总碱值是指在规定条件下, 中和 $1 \mathrm{~g}$ 试 样中全部碱组分所需酸的量, 以相当的氢氧化钾的 毫克数表示. 基础油为 MCT-10 型矿物油, 其硫含量 不超过 $0.2 \%$. 添加剂在基础油中的浓度为, ZDDP $1.2 \%$, 金属清净剂 $2.0 \%$ 或 $6.0 \%$ (均为质量分数).

\section{2 摩擦膜的制备}

摩擦膜在英国 Phoenix Tribology 公司生产的 Cameron-Plint Model TE-77 往复磨损试验机上制备,
采用销-盘线接触形式, 试盘直径为 $19 \mathrm{~mm}$, 厚度为 $5 \mathrm{~mm}$, 销直径为 $6 \mathrm{~mm}$, 长度为 $11 \mathrm{~mm}$, 试盘和销材 料均为 52100 钢. 使用 $3 \mu \mathrm{m}$ 金钢石砂纸将试盘抛 光至镜面, 后使用分析纯石油醚超声清洗后空气干 燥备用. 实验条件: 温度 $100{ }^{\circ} \mathrm{C}$, 载荷 $220 \mathrm{~N}$, 频率 25 $\mathrm{Hz}$, 摩擦时间 $0.5 \mathrm{~h}$ 和滑动距离 $7 \mathrm{~mm}$. 摩擦结束后, 钢盘用分析纯石油醚超声清洗, 空气干燥后供 XANES 分析.

\section{3 数据处理和分析}

磷、硫、钙和碳的 X 射线吸收谱收集于同步加 速器中心(University of Wisconsin, Madison). 通过双 晶单色器(DCM)产生能量范围 1500-4000 eV(分辨 率约为 $0.8 \mathrm{eV}$ ) 的 $\mathrm{X}$ 射线来获得硫和磷的 $K$-边光谱; 通过 Grasshopper 软 X 射线光束产生能量范围 70$900 \mathrm{eV}$ (分辨率小于 $0.2 \mathrm{eV}$ ) 的 $\mathrm{X}$ 射线采集硫和磷的 $L$-边光谱; 通过球型光栅单色器(SGM) 来获得碳的 $K$-边光谱(分辨率小于 $0.05 \mathrm{eV}$ ) 和钙的 $L$-边光谱. 光 子吸收光谱通过 TEY 和 FY 两种模式记录, 分别用 来提供表面/次表面和本体上的化学信息. $L$-边光谱 数据首先用标准化合物 ZDDP 在 $164.0 \mathrm{eV}$ 处的峰 进行校正, 而 $K$-边光谱数据用标准化合物硫化锌在 $2473.0 \mathrm{eV}$ 处的峰进行校正. 每个样品经过至少两次 扫描并相加, 之后均除去背景得谱图.

\section{2 实验结果}

\section{1 摩擦膜中磷的表征}

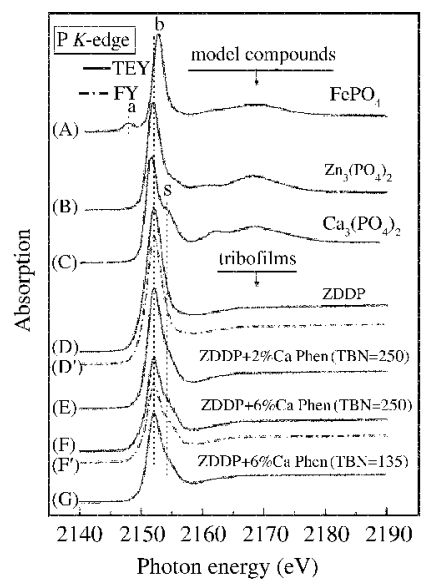

图 1 模型化合物及二烷基二硫代磷酸锌(ZDDP)和酚钻 复配使用形成摩擦膜的磷 $K$-边 XANES 谱图

Fig.1 P K -edge XANES spectra of model compounds and tribofilms generated from ZDDP and its combination with Ca phenate (Phen) ZDDP: zinc dialkyldithiophosphate; TBN: total base number; TEY: total electron yield; FY: fluorescence yield 
图 1 是单独 ZDDP 和 ZDDP 与两种酚钙复配 润滑下摩擦膜的磷的 $K$-边 XANES 谱图(谱线 D-G), 为了比较, 图中同时给出几种标准化合物的磷的 $K$ 边 XANES 谱图 (谱线 A-C). 可以看出, 对所有的摩 擦膜, 磷的 $K$-边 XANES 谱图均有一个吸收峰. 谱线 D 来自单独 ZDDP 润滑下的摩擦膜, 与标准化合物 对比, 吸收峰的位置与磷酸锌(谱线 B)相同, 而与磷 酸铁(谱线 A)有一定的差别, 这说明在 XANES 技术 的检测灵敏度范围内 (对 $K$-边约为 $10^{-4}$ ), 表面上存在 着磷酸锌而没有磷酸铁. 谱线 $\mathrm{E}-\mathrm{G}$ 来自于ZDDP 和 酚钙复配使用时产生的摩擦膜, 除了一个强的吸收 峰外, 还明显存在一个肩峰, 位置与磷酸钲(谱线 C) 的肩峰位置相一致, 这说明摩擦膜中存在着一定数 量的磷酸钙. 此外, 肩峰的大小明显与酚钙总碱值的 高低和使用的浓度有关. 比较谱线 $\mathrm{E}$ 和 $\mathrm{F}$ 可以看出, 对高碱值酚钙- 2 , 与 ZDDP 复配使用时酚钙的浓度 越高, 肩峰越大, 即在摩擦膜中存在更多的磷酸钙; 同样比较谱线 $\mathrm{G}$ 和 $\mathrm{F}$, 在相同的酚钙浓度 $(6 \%)$ 下, 碱 值较低的酚钙- 1 与 ZDDP 复配使用形成的摩擦膜 (谱线 $\mathrm{G}$ )中仍可观察到磷酸钙的存在, 但数量明显少 于相应的较高碱值酚钙-2 与 ZDDP 复配使用形成 的摩擦膜(谱线 F).

XANES 谱图中 $L$-边特征峰的产生是由于电子 从 $2 p$ 轨道跃迁至一个空的 $s$ 或 $d$ 轨道, 所需能量较 低, 具有更高的分辨率; 而 $K$-边 XANES 谱图由于 $1 s$ 上的电子跃迁至一个 $p$ 轨道, 扫描时需要很高的 能量. TEY 模式下 $L$-边探针厚度约 $5 \mathrm{~nm}$, 而 $K$-边探 针的厚度约 $50 \mathrm{~nm}$, 因此 $L$-边具有更高的表面敏感 性, 可以提供比 $K$-边更细致的表面化学信息.

图 2 是单独 ZDDP 和 ZDDP 与酚钙复配润滑 下摩擦膜的磷的 $L$-边 XANES 谱图. 谱线 B 来自于 单独 ZDDP 润滑下形成的摩擦膜, 与标准化合物的 谱线相比, 峰 $\mathrm{c}$ 的位置与聚磷酸锌(谱线 $\mathrm{A}$ )相同, 说 明表面上存在磷酸锌. 当 ZDDP 与酚钻复配使用时 (谱线 C-E), 我们观察到谱图中吸收峰的位置向低 能量方向发生了转移, 同时出现了肩峰, 峰的位置和 磷酸钻(谱线 F)的峰位十分接近. 这再一次证实了磷 的 $K$-边 XANES 谱(图 1)得到的结果, 当 ZDDP 与酚 钙复配使用时, 摩擦膜表面上存在着磷酸钻. 同样肩 峰的大小与酚钙的总碱值和在基础油中的浓度有 关, 总碱值越大, 浓度越大, 肩峰就越大, 也即表面中 磷酸钙的量就越多. 如果仔细研究 ZDDP 与 $6 \%$ 酚 钙-2 表面膜(谱线 D), 其肩峰的大小已超过标准化

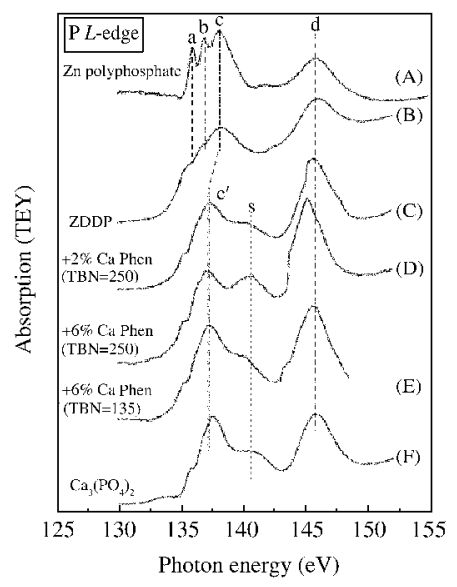

图 2 模型化合物及 ZDDP 和酚钙复配使用形成摩擦膜的 磷 $L$-边 XANES 谱图

Fig.2 P L-edge XANES spectra of model compounds and tribofilms generated from ZDDP and its combination with Ca phenate

合物磷酸钙(谱线 F) 相应的肩峰, 这说明除磷酸钻 外, 其他类型的含钲化合物可能存在于摩擦膜中. 在 下一节中利用碳 $K$-边和钙 $L$-边的 XANES 谱对此进 行研究.

在以前的研究 ${ }^{[20]}$ 中曾发现, 在磷的 $L$-边 XANES 谱图中, 峰 $\mathrm{c}$ 对峰 $\mathrm{a}$ 的相对强度比可用来表征聚磷 酸锌的链长高低, 其数值越大, 聚磷酸锌的链长越 长, 事实上, 聚磷酸锌链长极大地影响摩擦膜的抗磨 性能, 链长越长, 抗磨性能越好. 从图 2 可以看出, 当 ZDDP 与酚钙复配使用时, 摩擦膜中生成磷酸盐的 链长明显减小.

\section{2 摩擦膜中碳和钙的表征}

碳元素可能以有机碳和无机碳两种形式存在于 摩擦膜中. 有机碳来源于基础油、添加剂本身在表面 的吸附等, 而无机碳主要来源于酚钙中的过碱组分 (碳酸钙). 这里我们利用 SGM 单色器收集了单独 ZDDP 和 ZDDP 与酚钙复配使用下碳的 $K$-边和钻的 $L$-边的谱图, 其结果示于图 3 中.

标准化合物羰酸钙(谱线 B) 具有一些特征峰. 在碳的 $K$-边谱图中有两个强的吸收峰, 在钙的 $L$-边 存在典型的双吸收峰. 谱线 $\mathrm{A}$ 是来自于用来承载羰 酸钙的导电胶带, 在碳的 $K$-边谱图中的吸收峰可认 为来自于有机碳, 在钙的 $L$-边无吸收峰. 谱线 $\mathrm{C}$ 来 自于单独 ZDDP 润滑下的摩擦膜, 碳的 $K$-边谱图中 的吸收峰可认为来自于有机碳, 同样在钙的 $L$-边无 峰. 谱图 D-F 来自于 ZDDP 与酚钙复配润滑下形成 的摩擦膜, 其中谱图 $\mathrm{D}$ 和谱图 $\mathrm{E}$ 的曲线形状十分接 近, 在碳的 $K$-边谱图中有一个吸收峰, 可以认为来自 


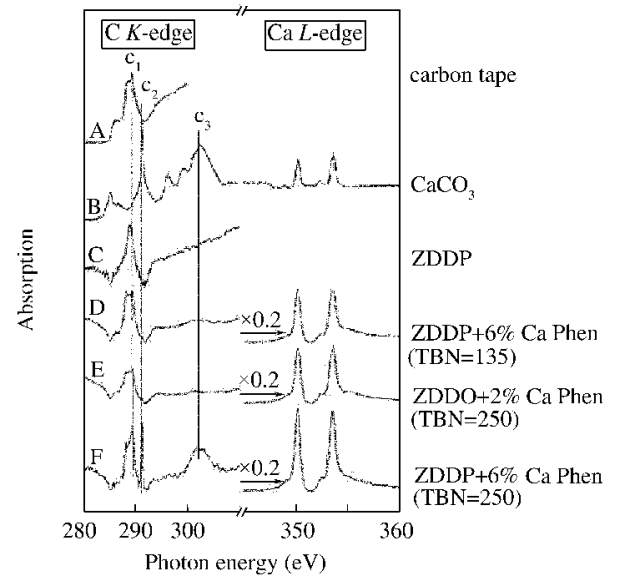

图 3 模型化合物及 ZDDP 和酚钙复配使用形成摩擦膜的 碳 $K$-边和钙 $L_{2,3}$-边 XANES 谱图

Fig.3 C $K$-edge and $C a L_{2,3}$-edge XANES spectra of model compounds and tribofilms generated from

ZDDP and its combination with Ca phenate

于有机碳, 在钙的 $L$-边谱图可以看见典型钻的双吸 收峰, 这再次说明在摩擦膜中存在着含钻的化合物, 根据前面磷的 $K$-边和 $L$-边的 XANES 谱图的分析结 果可以认定为磷酸钻. 谱图 $\mathrm{F}$ 来自于 ZDDP与 $6 \%$ 的较高碱值酚钙- 2 复配润滑下形成的摩擦膜, 除在 碳的 $K$-边谱图中观察到来自于有机碳的吸收峰外, 还存在另一个尖锐的吸收峰, 其位置与标准化合物 碳酸钲(谱线 B)相吻合. 同样, 在钻的 $L$-边谱图中可 观察到典型钙的双吸收峰, 这说明表面上存在着碳 酸钙, 这可能是因为在滑动过程中高碱值酚钙的胶 体结构被破坏, 释放出的碳酸钙颗粒沉积在摩擦膜 中. 但如果比较谱线 B 和谱线 $\mathrm{F}$ 中碳的 $K$-边谱图中 $\mathrm{c}_{2}$ 峰和钻的 $L$-边谱图中双峰的相对强度可以看出, ZDDP 与 $6 \%$ 的高碱值酚钙 -2 复配润滑下形成的摩 擦膜除含有碳酸钻外, 还有磷酸钙.

\section{3 摩擦膜中硫的表征}

图 4 是单独 ZDDP 及 ZDDP 与酚钙 -2 复配润 滑时摩擦膜硫的 $K$-边 TEY 光谱. 谱线 D 来自于单 独 ZDDP 润滑下形成的摩擦膜, 其吸收峰的位置和 标准化合物 $\mathrm{ZnS}$ (谱线 C) 相同, 说明 ZDDP 在滑动 过程中经历了摩擦化学反应, 在表面形成了硫化锌 膜. ZDDP 与 $2 \%$ 酚钙- 2 复配使用时形成的摩擦膜的 结构(谱线 E) 与单独 ZDDP 形成的摩擦膜(谱线 D) 十分相似, 而 ZDDP 与 $6 \%$ 酚钙-2 复配使用时形成 的摩擦膜(谱线 F) 则不同, 谱图除了 $\mathrm{ZnS}$ 的吸收峰 外, 还有一个强度更大的吸收峰, 与标准化合物相比 后可确认为四价的亚硫酸盐. 由于在单独 ZDDP 及 ZDDP 与 $2 \%$ 酚钙-2 复配使用产生的摩擦膜的谱线

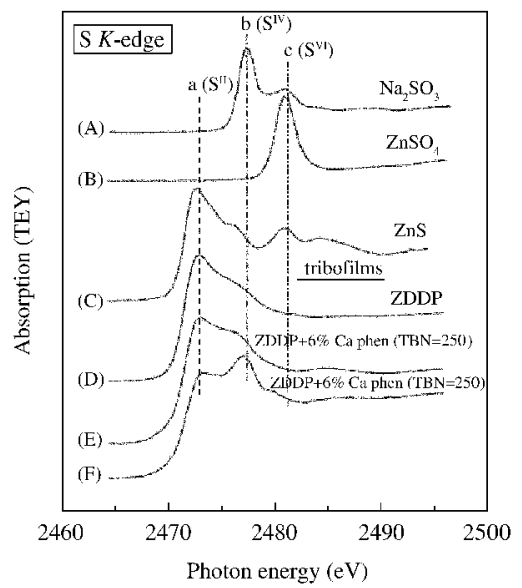

图 4 模型化合物和 ZDDP 及其与酚钙复配使用形成摩擦 膜的硫 $K$-边 XANES 谱图

Fig.4 S K-edge XANES spectra of model compounds and tribofilms generated from ZDDP and its combination with Ca phenate

中均未发现此峰, 可以认为亚硫酸盐的吸收峰是来 自于酚钙-2 在摩擦过程中的氧化造成的, 这在以前 的研究中证实过这一结果 ${ }^{[19]}$. 当 ZDDP 与 $2 \%$ 酚钙 -2 复配使用时, ZDDP 本身的抗氧化性能阻止了酚钙 的氧化. 当酚钙- 2 的浓度增加到 $6 \%$ 时, ZDDP 并不 能完全阻止酚钙- 2 的氧化, 部分酚钙- 2 被氧化生成 亚硫酸盐, 此时酚钙-2 的胶体结构可能被破坏, 释 放出的碳酸钙颗粒则沉积在摩擦膜表面. 这符合上 述碳的 $K$-边和钙的 $L$-边的分析结果.

\section{4 摩擦膜的厚度}

在以前的研究中, 我们利用磷的 $K$-边 $\mathrm{FY}$ 曲线 的强度可以得到摩擦膜中磷成分的质量厚度(或面 密度 $)^{[15]}$. 如果忽略摩擦膜中碳酸钻的影响, 可以比 较酚钲对摩擦膜厚度的影响, 其结果见表 1 . 可以看 出单独 ZDDP 产生最厚的摩擦膜, 加人酚钙后, 摩 擦膜的厚度显著降低, 降低的程度取决于酚钙的浓 度和总碱值. 总碱值越大, 使用的酚钙浓度越大, 产生 的摩擦膜厚度越小. 实验结果证实了Willermet 等 ${ }^{[2]]}$ 使用俄歇电子能谱和表面形貌仪得到的结果, 当

表 1 ZDDP 和酚钙复配使用形成摩擦膜质量厚度

Table 1 Mass thickness of tribofilms and antiwear properties of ZDDP and its combination with calcium phenates

\begin{tabular}{lcc}
\hline Complex & $\begin{array}{c}\text { P areal density } \\
\left(10^{16} \text { atoms }\right)\end{array}$ & $\begin{array}{c}\text { Wear scar width } \\
(\mathrm{mm})\end{array}$ \\
\hline base oil & 6.9 & 0.32 \\
ZDDP & 3.9 & 0.06 \\
ZDDP+6\%Ca Phen (TBN=135) & & 0.16 \\
ZDDP+2\%Ca Phen (TBN=250) & 1.2 & 0.16 \\
ZDDP+6\%Ca Phen (TBN=250) & & 0.18 \\
\hline
\end{tabular}


ZDDP 单独使用时, 摩擦膜厚约为 $53 \mathrm{~nm}$, 加人酚钙 后减小到约 $40 \mathrm{~nm}$.

\section{5 抗磨性能}

利用栓盘型试验机评价了 ZDDP 及 ZDDP 与 酚钙复配体系的抗磨性能, 其结果示于表 1 中. 抗 磨性能是用栓试样的磨斑直径来表示, 其值越小, 抗磨性能越好. 从表 1 中可见, 与基础油相比, ZDDP 具有明显的抗磨作用; 加人酚钙后, ZDDP 的 抗磨性能变差, 尤其以高浓度和高碱值的酚钻和 ZDDP 的复配体系为甚.

\section{3 结果与讨论}

ZDDP 在钢钢摩擦副中的作用机制可归结为其 在滑动过程中于表面反应生成的含磷、硫和锌的摩 擦膜阻止了摩擦副的接触, 这层膜具有较低的剪切 系数, 从而降低了摩擦和磨损. 近年来, 人们利用不 同的表面分析手段详细研究了摩擦膜的表面结构. 利用 X 射线近边结构光谱发现, ZDDP 在滑动过程 中在接触表面发生复杂的物理化学反应, 在表面上 生成双层膜结构, 外层为长链的网状聚磷酸锌膜, 内 层为短链的聚磷酸锌膜, 其中外层长链的聚磷酸锌

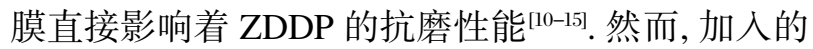
酚钙完全改变了 ZDDP 形成摩擦膜的结构, 聚磷酸 锌双层膜结构被破坏, 膜中含有酚钙中的钙离子, 其 结构以短链磷酸钙为主, 对高碱值的酚钙, 高浓度 时, 在摩擦膜同时观测到碳酸钙颗粒的沉积. 摩擦膜 结构的改变直接影响了其抗磨性能.

酚钙和 ZDDP 复配使用时, 摩擦膜中磷酸钙的 生成可能是聚磷酸锌与酚钙中钙离子发生离子交 换反应而产生的, 其过程可用 Peterson 的软硬酸碱 理论来解释. $\mathrm{PO}_{4}^{3-}$ 为典型的强碱, 而 $\mathrm{Ca}^{2+}$ 的酸性比 $\mathrm{Zn}^{2+}$ 强, 也就是说 $\mathrm{Ca}^{2+}$ 可能置换磷酸锌中的 $\mathrm{Zn}^{2+}$ 形成 更稳定的磷酸钲.

关于磷酸锌/钙复合摩擦膜抗磨性能的研究未见 文献报道, 从目前的实验结果可以看出其抗磨性能 比单纯的磷酸锌差, 系统探讨磷酸锌/钲复合摩擦膜 纳米机械性能的研究将是我们下一步工作的重点.

\section{4 结 论}

用磷的 $K$-边和 $L$-边、碳的 $K$-边、钙的 $L$-边和硫 的 $K$-边 XANES 谱表征 ZDDP 和酚钙复配体系润滑 下形成的摩擦膜的表面化学. 结果表明, 烷基特性 酚盐存在时, 单独 ZDDP 形成的双层膜结构被破坏,
摩擦膜中存在着一定数量的磷酸钙; 对高碱值的酚 钙, 高浓度下使用时, 其胶体结构可能被破坏, 碳酸 钻可沉积在摩擦膜表面上.

致谢：本文工作是在加拿大西安大略大学化学系 M. Kasrai 博士和 G. M. Bancroft 教授的直接指导和帮助下完成的, 在 此表示诚挚的谢意.

\section{References}

1 Huang, W. X. Application guide of lubricant additives. Beijing: SinoPec Press, 2003 [黄文轩. 润滑剂添加剂应用指南. 北京: 中 国石化出版社, 2003]

2 Spikes, H. Tribol. Lett., 2004, 17: 469

3 Nicholls, M. A.; Do, T.; Norton, P. R.; Kasrai, M.; Bancroft, G. M. Tribol. Int., 2005, 38: 15

4 Morina, A.; Green, J. H.; Neville, A.; Priest, M. Tribol. Lett., 2003, 15: 443

5 Minfray, C.; Le Mogne, T.; Lubrecht, A. A.; Martin, J. M. Tribol. Lett., 2006, 21: 67

6 Minfray, C.; Martin, J. M.; de Barros, M. I.; Le Mogne, T.; Kersting, R.; Hagenhoff, B. Tribol. Lett., 2004, 17: 351

7 Yamaguchi, E. S. Tribol. Int., 2003, 36: 727

8 Minfray, C.; Martin, J. M.; Esnouf, C.; Le Mogne, T.; Kersting, R.; Hagenhoff, B. Thin Solid Films, 2004, 447-448: 272

9 Stoehr, J. NEXAFS spectroscopy. Berlin: Springer, 1996

10 Pereira, G.; Lachenwitzer, A.; Kasrai, M. Tribol. Lett., 2007, 26: 103

11 Nicholls, M. A.; Do, T.; Norton, P. R.; Nicholls, M. A.; Kasrai, M.; Bancroft, G. Tribol. Int., 2005, 38: 15

12 Varlot, K.; Kasrai, M.; Brancroft, G. M.; Yamaguchi, E. S.; Ryason, P. R. Wear, 2001, 249: 1029

13 Zhang, Z.; Kasrai, M.; Bancroft, G. M.; Yamaguchi, E. S. Tribol. Lett., 2003, 15: 377

14 Yamaguchi, E. S.; Zhang, Z.; Kasrai, M.; Bancroft, G. M. Tribol. Lett., 2003, 15: 385

15 Fuller, M. L. S.; Rodriguez, L.; Massoumi, G. R.; Lennard, W. N.; Kasrai, M.; Bancroft, G. M. Tribol. Lett., 2000, 8: 187

16 Yi, H. L.; Wu, H.; Zeng, X. Q.; Ren, T. H. Acta Phys. -Chim. Sin., 2006, 22: 1473 [易红玲, 吴 华, 曾祥琼, 任天辉. 物理化学学 报, 2006, 22: 1473]

17 Wu, H.; Fang, K. Z.; Li, J.; Ren, T. H.; Ji, K. Y. Acta Phys. -Chim. Sin., 2007, 23: 911 [吴 华, 范开忠, 李 晶, 任天辉, 及开元 物理化学学报, 2007, 23: 911]

18 Ma, H. B.; Li, J.; Yu, Y.; Zuo, G. Z.; Ren, T. H. Acta Phys. -Chim. Sin., 2008, 24: 799 [马海兵, 李 晶, 於 薏, 左光之, 任天辉. 物理化学学报, 2008, 24: 799]

19 Wan, Y.; Kasrai, M.; Bancroft, G. M. Chin. J. Vac. Sci. Technol., (in press) [万 勇, Kasrai, M., Bancroft, G. M. 真空科学与技术 学报, 印刷中]

20 Yin, Z.; Kasrai, M.; Bancroft, G. M.; Tan, K. H.; Feng, X. H. Phys Rev. B, 1995, 51: 742

21 Willermet, P. A.; Carter, R. O.; Schmitz, P. J.; Everson, M.; Scholl, D. J.; Weber, W. H. Lub. Sci., 1997, 9: 325 